\title{
Determinantes sociais de alterações fonoaudiológicas
}

\author{
Social determinants of speech-language disorders
}

\author{
Albanita Gomes da Costa de Ceballos ${ }^{1}$, Carla Cardoso ${ }^{2}$
}

\begin{abstract}
RESUMO
Objetivo: Verificar a associação entre fatores sócio-econômicos e alterações fonoaudiológicas auto-referidas. Métodos: Este estudo foi realizado por meio de entrevistas domiciliares com 543 adultos residentes na cidade de Salvador (BA). Resultados: Os resultados mostraram associações positivas e estatisticamente significantes entre escolaridade e queixas de alterações auditivas ( $\mathrm{RP}=1,48$ com IC95\% 1,22-1,81) e de escolaridade e queixas de linguagem ( $\mathrm{RP}=1,69$ com IC95\% 1,36-2,11). Também foram encontradas associações entre renda e queixas de alterações de motricidade oral ( $\mathrm{RP}=1,34$ com IC95\% 1,13-1,60) e renda com queixas vocais ( $\mathrm{RP}=1,24$ com IC95\% 1,08-1,44). Conclusão: Conclui-se que condições adversas de vida relacionam-se a alta prevalência de distúrbios fonoaudiológicos em comunidades.
\end{abstract}

Descritores: Fatores epidemiológicos; Doença; Condições sociais; Meio ambiente; Saúde pública

\section{INTRODUÇÃO}

Condições sociais são determinantes dos estados de saú$\mathrm{de}^{(1-4)}$. Fatores sociais afetam as condições biológicas individuais, os comportamentos de risco, as exposições ambientais e o acesso a recursos de promoção à saúde.

Pessoas com diferentes níveis de escolarização e renda, diferentes tipos de ocupação e que moram em localidades sem infra-estrutura adequada, têm diferenças na expectativa de vida ${ }^{(5)}$. Historicamente, o baixo status sócio-econômico tem sido referido como associado com altas razões de morbidade como, por exemplo, em casos de doenças infecciosas ${ }^{(6)}$. Mais recentemente, estudos apontam associações entre determinantes socioeconômicos e outras condições de saúde como peso ao nascer ${ }^{(7-8)}$, doenças respiratórias ${ }^{(8)}$, duração do aleitamento materno ${ }^{(8-9)} \mathrm{e}$ hábitos orais inadequados ${ }^{(10)}$.

Conhecer os determinantes socioeconômicos das doenças é importante para poder pensar em estratégias de enfrentamento e melhoria das condições de saúde da população ${ }^{(11)}$. A teoria ecossocial apresentada por Krieger ${ }^{(11)}$, aponta que o corpo evidencia o ambiente em que vive por intermédio dos seus padrões de saúde, de doença, de incapacidade e de morte.

Pessoas com piores condições sócio-econômicas têm me-

Trabalho realizado no Curso de Fonoaudiologia do Centro Universitário Jorge Amado - UNIJORGE - Salvador (BA), Brasil.

(1) Doutora, Professora do Curso de Fonoaudiologia do Centro Universitário Jorge Amado - UNIJORGE e da Universidade do Estado da Bahia - UNEB - Salvador (BA), Brasil.

(2) Doutora, Professora do Curso de Fonoaudiologia do Centro Universitário Jorge Amado - UNIJORGE - Salvador (BA), Brasil.

Endereço para correpondência: Albanita Gomes da Costa de Ceballos. R. Almirante Barroso, 248, BM 202, Rio Vermelho, Salvador (BA), Brasil, CEP: 41950-350. E-mail: albanitagomes@msn.com

Recebido em: 24/7/2008; Aceito em: 25/4/2009 nor expectativa de vida e mais doenças ${ }^{(5)}$. Além disto, fatores como estresse, condições de vida durante os primeiros anos de idade, exclusão social, desemprego e outros, também contribuem para o adoecimento.

Estudos na área da Fonoaudiologia mostram que existe relação entre condição sócio-econômica e hábitos orais capazes de promover alterações fonoaudiológicas, otorrinolaringológicas e odontológicas ${ }^{(12)}$. $\mathrm{O}$ aspecto sócio-econômico está relacionado tanto ao crescimento de bebês quanto ao desenvolvimento da sua linguagem oral, desenvolvimento físico e motor ${ }^{(13-14)}$. Além disso, a perda auditiva mostra-se mais frequente em crianças com menor poder aquisitivo ${ }^{(15)}$. A paracoccidioidomicose, micose causada por fungo, que leva a sintomas de disfonia também aparece na literatura como relacionada a fatores sócio-econômicos como ocupação e renda ${ }^{(16)}$.

Diante do exposto, o objetivo deste estudo foi verificar a associação entre fatores sócio-econômicos e alterações fonoaudiológicas auto-referidas. $\mathrm{O}$ mesmo se justifica pela precariedade de literatura sobre o tema e pela relevância do conhecimento para a tomada de decisões de cunho político para o desenvolvimento de práticas preventivas focalizada na realidade do indivíduo em risco.

\section{MÉTODOS}

\section{Área do estudo}

Este estudo foi realizado em uma comunidade de Salvador (BA). Esta comunidade nasceu a partir de uma ocupação urbana e, posteriormente, consolidou-se como bairro. O bairro atualmente conta com aproximadamente 11 mil domicílios e uma população de 65 mil habitantes. O IDH (Índice de 
Desenvolvimento Humano) é considerado um dos piores da capital baiana $(0,664)$ e a esperança de vida é de $62,08 \operatorname{anos}^{(17)}$.

\section{População do estudo e coleta de dados}

O estudo foi desenvolvido nos moldes de um estudo transversal analítico dentro da disciplina Práticas em Saúde Pública de um curso de Fonoaudiologia, no primeiro semestre do ano de 2007.

Sendo um estudo exploratório e diante da ausência de dados de prevalência das alterações fonoaudiológicas estudadas, não se realizou cálculo amostral.

Foram selecionadas as ruas residenciais no entorno da unidade de saúde local. A partir daí, foram considerados eletivos ao estudo todos os residentes maiores de 18 anos de idade, excluindo-se apenas os que não se encontravam em aparente condição de responder aos questionamentos (pessoas em estado de embriagues, idosos com sinais de demência* ou indivíduos com deficiência auditiva incapacitante para compreensão da linguagem oral).

Foram visitados 163 domicílios e responderam aos questionários 543 indivíduos adultos (4,73\% de perdas e recusas). As visitas domiciliares foram realizadas por alunos previamente treinados. Os indivíduos pesquisados responderam a um questionário desenvolvido para este estudo, com base nas queixas mais freqüentes encontradas na clínica fonoaudiológica (Anexo 1). O mesmo contém questões sócio-econômicas, de saúde geral e de saúde fonoaudiológica. Este último bloco contém quatro sub-blocos referentes às quatro grandes áreas de atuação da Fonoaudiologia: audição, linguagem, motricidade orofacial e voz.

\section{Considerações éticas}

O estudo foi aprovado por Comissão de Ética em Pesquisa do Centro Universitário Jorge Amado - UNIJORGE (parecer número 0352008). Os sujeitos foram informados sobre os objetivos e o método do estudo e assinaram um termo de consentimento livre e esclarecido. A pesquisa não representou risco físico ou moral aos sujeitos.

\section{Análise de dados}

Os dados coletados foram digitados no programa Epi-Info 6.02 e analisados no programa SPSS versão 9.0. Inicialmente foram verificadas as frequências de cada variável com o objetivo de conhecer a distribuição da população e fazer sua descrição. As variáveis contínuas foram categorizadas ou dicotomizadas e novamente analisadas quanto à frequência. Em seguida, realizou-se a análise bivariada para constatar possíveis associações entre as variáveis sócio-demográficas e cada possível desfecho: (a) queixa de alteração de audição, (b) queixa de alteração de linguagem, (c) queixa de alteração de motricidade e (d) queixa de alteração na voz. Nesta avaliação são apresentados os valores da razão de prevalência e seus respectivos intervalos de confiança (IC95\%).

\section{RESULTADOS}

A análise descritiva mostrou que a população se caracteriza por ser formada por uma maioria de mulheres $(72,9 \%)$, com idade acima de 30 anos $(51,9 \%)$ e escolaridade fundamental $(58,9 \%)$. A cor da pele auto-referida pela maioria foi negra ou mulata $(71,6 \%)$. Quanto à renda média mensal do domicílio, $57,5 \%$ dos entrevistados afirmaram que é menor ou igual a um salário mínimo. Em 63,2\% dos domicílios residem menos de quatro pessoas e $73 \%$ dos sujeitos estavam desempregados no momento da coleta de dados. Quando questionados sobre a nota atribuída a sua saúde, quase $47 \%$ dos entrevistados responderam um valor menor do que cinco. Sobre as queixas de alterações fonoaudiológicas, 70,3\% afirmou ter queixa auditiva, $59,3 \%$ ter queixa vocal, $48,3 \%$ ter queixa de linguagem e $34,6 \%$, queixa de motricidade** (Tabelas 1 e 2).

Tabela 1. Descrição da população segundo variáveis sócio-demográficas

\begin{tabular}{|c|c|c|}
\hline Variável & $\mathrm{N}$ & $\%$ \\
\hline \multicolumn{3}{|l|}{ Sexo } \\
\hline Masculino & 147 & 27,1 \\
\hline Feminino & 396 & 72,9 \\
\hline \multicolumn{3}{|l|}{ Idade } \\
\hline Até 30 anos & 261 & 48,1 \\
\hline 30 ou mais anos & 282 & 51,9 \\
\hline \multicolumn{3}{|l|}{ Escolaridade } \\
\hline Médio ou mais & 219 & 40,3 \\
\hline Fundamental ou menos & 320 & 58,9 \\
\hline \multicolumn{3}{|l|}{ Cor da pele } \\
\hline Outras & 154 & 28,4 \\
\hline Negro/mulato & 389 & 71,6 \\
\hline \multicolumn{3}{|l|}{ Renda } \\
\hline Mais de $1 \mathrm{SM}$ & 231 & 42,5 \\
\hline Até $1 \mathrm{SM}$ & 312 & 57,5 \\
\hline \multicolumn{3}{|c|}{ Número de pessoas por domicílio } \\
\hline Até 4 & 343 & 63,2 \\
\hline Mais de 4 & 200 & 36,8 \\
\hline \multicolumn{3}{|l|}{ Emprego } \\
\hline Sim & 147 & 27,1 \\
\hline Não & 396 & 72,9 \\
\hline \multicolumn{3}{|l|}{ Nota atribuída a saúde } \\
\hline Mais de 7 & 235 & 43,3 \\
\hline 5 a 7 & 55 & 10,1 \\
\hline Menos de 5 & 253 & 46,6 \\
\hline
\end{tabular}

Legenda: $\mathrm{N}=$ número de pesquisados; \% = valor percentual; $\mathrm{SM}=$ salário mínimo

\footnotetext{
* Dificuldade de orientação espaço-temporal, problemas com o raciocínio abstrato incapacidade de julgar situações, problema com o vocabulário, perda da iniciativa - passividade ${ }^{(18)}$

** Valores percentuais não passíveis de somatório. Os indivíduos poderiam referir queixa em mais de uma das áreas.
} 
Tabela 2. Descrição da população segundo queixas de alterações fonoaudiológicas

\begin{tabular}{lcc}
\hline Variável & $\mathrm{N}$ & $\%$ \\
\hline Queixa de alteração de audição & 161 & 29,7 \\
Não & 382 & 70,3 \\
Sim & & \\
Queixa de alteração de linguagem & 281 & 51,7 \\
$\quad$ Não & 262 & 48,3 \\
Sim & & \\
Queixa de alteração de motricidade & 355 & 65,4 \\
$\quad$ Não & 188 & 34,6 \\
Sim & & \\
Queixa de alteração vocal & 221 & 40,7 \\
Não & 322 & 59,3 \\
$\quad$ Sim
\end{tabular}

Legenda: $\mathrm{N}$ = número de pesquisados; \% = valor percentual

A Tabela 3 apresenta as razões de prevalência e seus respectivos intervalos de confiança para um alfa igual a 5\%. A baixa escolaridade mostrou uma associação positiva e estatisticamente significante com as queixas de alteração auditiva e de linguagem. Já a renda igual ou menor que um salário mínimo mostrou-se associada de forma positiva e estatisticamente significante com as queixas de motricidade orofacial e vocal.

\section{DISCUSSÃO}

Este estudo mostrou a correlação entre os determinantes sociais, escolaridade, renda e emprego, com as queixas fonoaudiológicas.

O perfil da comunidade mostra uma maior proporção de pessoas do sexo feminino e sem emprego. Sobre esta informação, é preciso ressaltar que as visitas para entrevistas foram feitas nas segundas e terças-feiras, durante os períodos da manhã e da tarde. Nestes horários, é mais fácil encontrar mulheres do que homens, pois, segundo é ditado pela cultura vigente, é delas o dever de cuidar da casa e da família.

Também neste horário, é mais comum encontrar as pessoas que não estavam naquele momento, exercendo alguma atividade de trabalho remunerado. Caso as visitas fossem realizadas em dias e horários diferentes, possivelmente o perfil sofreria alterações.

Quando questionados sobre a sua saúde geral, observou-se ainda uma inconsistência na nota atribuída à saúde. Pessoas que referiam a presença de problemas como hipertensão arterial, diabetes e outros, por vezes, atribuíam altas notas a sua saúde. É possível refletir que fatores religiosos possam ter influenciado na resposta de alguns que, mesmo referindo má saúde, afirmavam que sua saúde poderia ser pior e, por isso, a consideravam digna de receber boa nota ao ser comparada à de outras pessoas.

Verificou-se que a baixa escolaridade está associada às queixas auditivas e de linguagem, ao passo que a baixa renda está associada a alterações da motricidade oral e vocal.

Estes resultados encontram respaldo na literatura consultada que mostra a relação da escolaridade e renda com baixo peso ao nascer e doenças infecciosas e respiratórias ${ }^{(6-8)}$. O baixo peso ao nascer é fator de risco para atrasos no desenvolvimento motor $^{(17)}$, para deficiência auditiva ${ }^{(18)}$ e para algumas doenças infecciosas ${ }^{(19)}$. Tais doenças infecciosas, por sua vez, influenciam nos problemas vocais ${ }^{(20-21)}$. Desta forma, é possível afirmar que as condições sociais de vida contribuem para o adoecimento.

\section{CONCLUSÃO}

O surgimento de alterações auditivas, de linguagem, de motricidade orofacial e vocal, bem como a prevalência das mesmas, é afetado pelo ambiente em que se vive. Sugere-se que o instrumento utilizado para a abordagem dos pesquisados seja validado em um estudo futuro.

Tabela 3. Razão de prevalência entre variáveis econômicas e sociais e queixa de alteração fonoaudiológicas

\begin{tabular}{|c|c|c|c|c|c|c|c|c|}
\hline \multirow[t]{2}{*}{ Variável } & \multicolumn{2}{|c|}{ Queixa auditiva } & \multicolumn{2}{|c|}{ Queixa de linguagem } & \multicolumn{2}{|c|}{ Queixa de motricidade } & \multicolumn{2}{|c|}{ Queixa vocal } \\
\hline & $\mathrm{RP}$ & IC95\% & $\mathrm{RP}$ & IC95\% & $\mathrm{RP}$ & IC95\% & $\mathrm{RP}$ & IC95\% \\
\hline \multicolumn{9}{|l|}{ Escolaridade } \\
\hline Médio ou mais & $0,73^{*}$ & $0,61-0,87$ & $0,70^{*}$ & $0,60-0,81$ & 1,03 & $0,89-1,20$ & 0,98 & $0,80-1,21$ \\
\hline Fundamental ou menos & $1,48^{*}$ & $1,22-1,81$ & $1,69^{*}$ & $1,36-2,11$ & 0,95 & $0,77-1,17$ & 1,01 & $0,80-1,21$ \\
\hline \multicolumn{9}{|l|}{ Renda } \\
\hline Mais de 1 salário mínimo & 0,96 & $0,87-1,19$ & 0,99 & $0,86-1,14$ & $0,69^{*}$ & $0,57-0,84$ & $0,72^{*}$ & $0,58-0,90$ \\
\hline Até 1 salário mínimo & 1,02 & $0,78-1,20$ & 1,00 & $0,82-1,22$ & $1,34^{*}$ & $1,13-1,60$ & $1,24^{\star}$ & $1,08-1,44$ \\
\hline \multicolumn{9}{|l|}{ Emprego } \\
\hline Sim & 0,82 & $0,60-1,13$ & 1,14 & $0,86-1,51$ & 0,81 & $0,61-1,07$ & 0,89 & $0,67-1,19$ \\
\hline Não & 1,06 & $0,96-1,18$ & 0,95 & $0,85-1,05$ & 1,08 & $0,96-1,21$ & 1,04 & $0,99-1,15$ \\
\hline
\end{tabular}

Legenda: RP = razão de prevalência; IC95\% = intervalo de confiança para alfa igual a 5

* Valor estatisticamente significante 


\begin{abstract}
Purpose: To verify the association between socioeconomic factors and self-referred speech-language disorders. Methods: This study was carried out through home interviews with 543 adults who lived in the city of Salvador (BA), Brazil. Results: Results showed positive statistically significant associations between level of education and hearing loss complaints (RP=1.48 with IC95\% 1.22-1.81), and between level of education and language complaints ( $\mathrm{RP}=1.69$ with IC95\% 1.36-2.11). Associations between income and oral motricity ( $\mathrm{RP}=1.34$ with IC95\% 1.13-1.60) and between income and vocal complaints ( $\mathrm{RP}=1.24$ with IC95\% 1.08-1.44) were also found. Conclusion: Adverse life conditions were related to high prevalence of speech-language disorders in communities.
\end{abstract}

Keywords: Epidemiologic factors; Disease; Social conditions; Environment; Public health

\title{
REFERÊNCIAS
}

1. Andrade SM, Soares DA, Matsuo T, Souza RKT, Mathias TAF, Iwakura MLH, Zequim MA. Condições de vida e mortalidade infantil no Estado do Paraná, Brasil, 1997/2001. Cad Saúde Pública = Rep Public Health. 2006;22(1):181-9.

2. Moreira MR, Cruz Neto O, Sucena LFM. Um olhar sobre condições de vida: mortalidade de crianças e adolescentes residentes em Manguinhos, Rio de Janeiro, Brasil. Cad Saúde Pública = Rep Public Health. 2003;19(1)161-73.

3. Teixeira MG, Meyer MA, Costa MCN, Paim JS, Silva LMV. Mortalidade por doenças infecciosas e parasitárias em Salvador - Bahia: evolução e diferenciais intra-urbanos segundo condições de vida. Rev Soc Bras Med Trop. 2002;35(5):491-7.

4. Buss PM, Pellegrini Filho A. A saúde e seus determinantes sociais. Phycis (Rio J). 2007;17(1):77-93.

5. Marmot M. Social determinants of health inequalities. Lancet. 2005;365(9464):1099-104

6. Smedley BD, Syme SL, editors. Promoting health: Intervention strategies from social and behavioral research. Washington DC: National Academy Press; 2000.

7. Barros FC, Victora CG. Epidemiologia da saúde infantil: um manual para diagnósticos comunitários. São Paulo: Hucitec/UNICEF; 1991.

8. Correia LL, Mcauliffe JF. Saúde materno-infantil. In: Rouquayrol MZ. Epidemiologia e saúde. 4a ed. Rio de Janeiro: Medsi; 1994. p. 315-42.

9. Monteiro CA. Saúde e nutrição das crianças de São Paulo: diagnóstico, contraste social. São Paulo: Hucitec; 1988.

10. Infante PF. An epidemiologic study of finger habits in preschool children, as related to malocclusion, socioeconomic status, race, sex, and size of community. ASDC J Dent Child. 1976;43(1):33-8.

11. Krieger N. A glossary for social epidemiology. J Epidemiol Community Health. 2001;55(10):693-700.

12. Cavassani VGS, Ribeiro SG, Nemr NK, Greco AM, Köhle J, Lehn CN. Hábitos orais de sucção: estudo piloto em população de baixa renda. Rev Bras Otorrinolaringol. 2003;69(1):106-10.
13. Andrade CRF. Fonoaudiologia em berçário normal e de risco. São Paulo: Editora Lovise; 1996.

14. Alves ACP, Taques MIM, Xavier C. Acompanhamento de crianças com história de prematuridade no ambulatório da Irmandade da Santa Casa de Misericórdia de São Paulo. In: Marchesan IQ, Zorzi JL, Gomes ICD. Tópicos em Fonoaudiologia. São Paulo: CEFAC: Lovise; 1996.

15. De Biase NG, Grellet M. Disacusias em crianças escolares de Ribeirão Preto. Rev Bras Otorrinolarongol. 1991;57(4):181-7.

16. Palheta-Neto FX, Moreira JS, Martins ACC, Cruz FJ, Gomes ER, Pezzin-Palheta AC. Estudo de 26 casos de Paracoccidioidomicose avaliados no Serviço de Otorrinolaringologia da Fundação Oswaldo Cruz (FIOCRUZ). Rev Bras Otorrinolaringol. 2003;69(5):622-7.

17. Halpern R, Barros FC, Horta BL, Victora CG. Desenvolvimento neuropsicomotor aos 12 meses de idade em uma coorte de base populacional no sul do Brasil: diferenciais conforme peso ao nascer e renda familiar. Cad Saúde Pública = Rep Public Health. 1996;12(Supl 1):73-8.

18. Segre CAM. Prevalência da perda auditiva em recém-nascidos de muito baixo peso. J Pediatr (Rio J). 2003;79(2):101-4.

19. Cecatto SB, Garcia RID, Costa KS, Abdo TRT, Rezende CEB, Rapoport PB. Análise das principais etiologias de deficiência auditiva em Escola Especial "Anne Sullivan". Rev Bras Otorrinolaringol. 2003;69(2):23540.

20. Pitrez PMC, Pitrez JLB. Infecções agudas das vias aéreas superiores: diagnóstico e tratamento ambulatorial. J Pediatr (Rio J). 2003;79(Supl 1):S77-86.

21. Prietsch SOM, Fischer GB, César JA, Lempek BS, Barbosa Júnior LV, Zogbi L, et al . Acute lower respiratory illness in under-five children in Rio Grande, Rio Grande do Sul State, Brazil: prevalence and risk factors. Cad Saúde Pública = Rep Public Health. 2008;24(6):1429-38. 
Anexo 1. Questionário sobre aspectos sócio-demográficos, condições de saúde geral e específica do campo da Fonoaudiologia

Entrevistador: Data:

\begin{tabular}{|c|c|c|c|c|}
\hline \multicolumn{5}{|c|}{ Aspectos sócio-demográficos } \\
\hline \multirow{2}{*}{\multicolumn{3}{|c|}{ 1. Número do questionário: _ - }} & 6. Está empregado atualmer & \\
\hline \multicolumn{2}{|c|}{ 2. Sexo: $(F)(M)$} & & (0) $\operatorname{Sim}(1)$ Não & \\
\hline \multicolumn{3}{|c|}{ 3. Idade: } & 7. Se sim, qual a ocupação: & \\
\hline \multicolumn{3}{|c|}{ 4. Cor auto-referida: } & 8. Qual a renda média mens & l da casa: \\
\hline (0) branco & \multicolumn{2}{|c|}{ (1) amarelo/asiático (2) pardo } & (0) Mais de $3.000,00$ & (1) Entre $1.000,00$ e $3.000,00$ \\
\hline (4) mulato & (5) negro & (6) outro & (2) Entre 500,00 e $1.000,00$ & (3) Entre 250,00 e 500,00 \\
\hline \multicolumn{3}{|c|}{ 5. Escolaridade: } & (4) Entre 100,00 e 250,00 & (5) Menos de 100,00 \\
\hline \multicolumn{3}{|r|}{ (1) superior em andamento } & 9. Quantas pessoas residem & na casa: \\
\hline \multicolumn{3}{|c|}{$\begin{array}{ll}\text { (2) superior incompleto } & \text { (3) médio completo }\end{array}$} & & \\
\hline \multicolumn{5}{|c|}{$\begin{array}{ll}\text { (4) médio em andamento } & \text { (5) médio incompleto }\end{array}$} \\
\hline \multicolumn{5}{|c|}{ 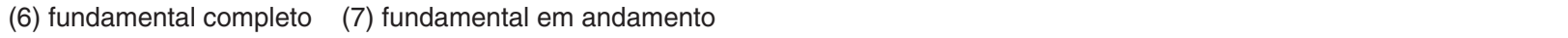 } \\
\hline \multicolumn{5}{|c|}{ (8) fundamental incompleto (9) Analfabeto } \\
\hline
\end{tabular}

\section{Aspectos gerais de saúde}

10. Doenças existentes:
(1) Diabetes
(2) Hipertensão arterial
(3) Câncer
(4) Aids
(5) Hanseníase
(6) Anemia falciforme

(7) Hepatite

(8) Sífilis

(9) Herpes

(10) Depressão (11) Outras:

11. História de má formação congênita ou síndromes na família:

(0) Não (1) Sim:

12. Qual a nota, de 0 a 10 , que dá a sua saúde: |___

\begin{tabular}{|c|c|c|}
\hline \multicolumn{3}{|l|}{ Aspectos específicos de saúde fonoaudiológica } \\
\hline Questões & Não (0) & $\operatorname{Sim}(1)$ \\
\hline \multicolumn{3}{|l|}{ 13.Tem alguma perda auditiva? } \\
\hline \multicolumn{3}{|l|}{ 14. Se está em uma festa ou ambiente com barulho, conversa com dificuldade? } \\
\hline \multicolumn{3}{|l|}{ 15. As pessoas reclamam que você vê televisão sempre com o volume muito alto? } \\
\hline \multicolumn{3}{|l|}{ 16. Geralmente não consegue ouvir bem o que as pessoas falam quando está ao telefone? } \\
\hline \multicolumn{3}{|l|}{ 17. Você observa que ao falar geralmente troca algumas letras por outras (ex: r por I)? } \\
\hline \multicolumn{3}{|l|}{ 18. Você observa que ao falar tem dificuldade em emitir alguns sons (ex: $r$ em Caruru)? } \\
\hline \multicolumn{3}{|l|}{ 19. Alguém já comentou ou você já percebeu que gagueja ao falar (explicar que é normal quando está nervoso)? } \\
\hline \multicolumn{3}{|l|}{ 20. Você sente dificuldade ao engolir algum alimento, água ou mesmo sua saliva? } \\
\hline \multicolumn{3}{|l|}{ 21. Alguém já comentou ou você já percebeu que quando engole algum alimento, água ou saliva faz barulho? } \\
\hline \multicolumn{3}{|l|}{ 22. Com muita freqüência tem engasgos ou sente dor ao tentar engolir um alimento, água ou saliva? } \\
\hline \multicolumn{3}{|l|}{ 23. Tem sensação de dor, ardor, corpo estranho ou aspereza na garganta (sem estar com resfriado)? } \\
\hline \multicolumn{3}{|l|}{ 24. Durante o dia sua voz vai piorando (sente ardor, tosse, ardor ou sensação de coceira)? } \\
\hline 25. No final do dia sua voz fica rouca, sai com dificuldade ou você perde a voz? & & \\
\hline
\end{tabular}

\section{Considerações finais}

26. Você já ouviu falar em Fonoaudiologia?
(0) $\mathrm{Sim}$
(1) Não

27. Você, ou alguém próximo, já procurou um fonoaudiólogo?

(0) Sim (1) Não

28. Você, ou alguém próximo, já fez tratamento com um fonoaudiólogo?

(0) Sim (1) Não

29. Algum outro profissional (ORL, pediatra, dentista, professor, etc) já disse que você, ou alguém próximo, deveria procurar um fonoaudiólogo?

(0) Sim: (1) Não

30. Você acha importante que os serviços de saúde tenham fonoaudiólogos para atender à população?
(0) Sim
(1) Não 